\title{
A Lazy Log-Keeping Mechanism for Comprehensive Global Garbage Detection on Amadeus
}

\author{
Sylvain Louboutin and Vinny Cahill \\ Distributed Systems Group, \\ Department of Computer Science, \\ Trinity College, Dublin 2, \\ Ireland.
}

E-mail: \{Sylvain.Louboutin,Vinny.Cahill\}@dsg.cs.tcd.ie

URL: http://www.dsg.cs.tcd.ie/

Fax: +353-1-6772204

\begin{abstract}
Global Garbage Detection (GGD) in object-oriented distributed systems requires that each application process maintains some information in support of GGD. Maintaining this information is known as log-keeping. In this paper we describe a low-overhead, log-keeping mechanism which proceeds lazily and avoids race conditions while nevertheless maintaining enough information for comprehensive GGD to take place.
\end{abstract}

KEY WORDS: Object-Oriented Distributed Systems, Comprehensive Global Garbage Detection

\section{Introduction}

Global Garbage Detection (GGD) in object-oriented distributed systems requires that each application process (conventionally called a mutator) maintains some information in support of GGD. Maintaining this information is known as log-keeping.

Log-keeping essentially entails keeping track of objects to which references have crossed site boundaries, and therefore become locally "alleged roots." Global Garbage Detection (GGD) consists in eventually identifying among these objects those which are not actually referenced remotely. It is up to the local GC to proceed with the actual collection of garbage objects.

The information maintained by the log-keeping mechanism constitutes a consistent, although not necessarily accurate, snapshot of the actual object graph, built incrementally. In Amadeus [Cahill et al., 1993] this snapshot is maintained as a set of logs, one log per-cluster.

We distinguish two strategies for log-keeping: eager and lazy. When an object reference crosses a site boundary, an eager log-keeping mechanism attempts to update the log maintained for the target object on the site where this object is located. This may involve additional control messages, e.g., when exchanging references of some third-party remote 


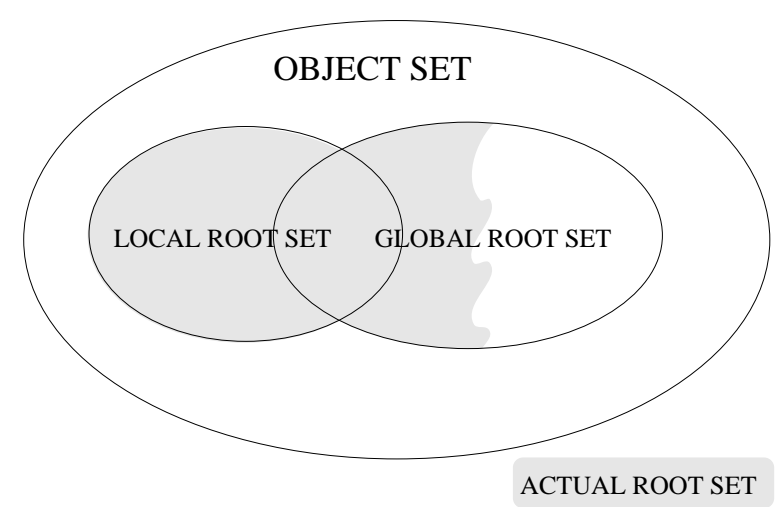

Figure 1: Object set, local root set, global root set and actual root set

object, and therefore potential race conditions between these messages and messages containing object references. These race conditions may jeopardize the consistency of the logs, leading to erroneously identifying a live object as garbage. Ensuring the consistency of the logs can therefore be both costly and complex when eager log-keeping is chosen. On the other hand, lazy log-keeping avoids additional control messages, without prejudice to the safety of the GGD.

Log-keeping is a necessary consequence of distribution, and does not dictate the nature of the GGD algorithm. However, the choice of strategy used by the GGD affects the nature and amount of information the log-keeping mechanism has to maintain. For instance, GGD approaches based on weighted reference counting [Bevan, 1987, Watson and Watson, 1987, Dickman, 1991] or reference listing [Plainfossé, 1994b] makes it possible to avoid eager logkeeping but are not intrinsically comprehensive.

This document describes a lazy log-keeping facility aimed at supporting comprehensive GGD on Amadeus [Cahill et al., 1993].

\section{System Model}

This section presents an abstract view of the underlying system which attempts not to be too specific about actual implementation details although reflecting the Amadeus [Cahill et al., 1993] model. It focuses on characteristics essential to the design of the logkeeping mechanism.

\section{$2.1 \quad$ Root Sets}

A site is a contiguous address space. Per-site GC is performed locally and independently of any other site. The root set for local GC consists of some local roots - the local root set i.e., objects arbitrarily designated as roots, plus some global roots - the global root set - i.e., objects alleged to be referenced from other (possibly remote) sites. The actual root set is made of objects, which although not necessarily reachable from a local root, are nevertheless alive; the union of the local root set and global root set is a superset of the actual root set as shown on Figure 1.

The actual root set cannot be efficiently known accurately at all times and a conservative approximation is used instead. This conservative approximation is the union of the local root set and the global root set, and is maintained jointly by mutators and the GGD algorithm. 
The mutator conservatively adds (write only) objects to the global root set as references to them cross site boundaries. The GGD purges the global root set to narrow it down to objects actually referenced from other sites. GGD is therefore decoupled from local garbage collection.

The invariant which the log-keeping mechanism must maintain with regards to root sets can be expressed as follows: the union of the local and global root sets is a superset of the actual root set of the local object graph.

\subsection{Objects}

An object is a contiguous portion of address space, whether on primary or secondary storage, potentially containing references to other objects. An object can be designated as being global, i.e., potentially known and invoked from a remote location, and/or persistent, i.e., may potentially outlive the thread of control that created it, as well as the context in which it was created. Conversely, an object can be local and/or volatile.

A persistent object should not hold references to any volatile object, so as to prevent the eventual occurrence of dangling references. All objects transitively referenced by a persistent object should eventually be made persistent.

\subsection{Clusters, Contexts and Containers}

A context is a transient address space. A cluster is a collection of one or more objects. Clusters of objects are the unit of mapping into contexts. Each context contains a set of clusters which may vary dynamically as clusters are created, mapped into or un-mapped from it. A cluster is mapped into at most one context at a time.

A cluster of persistent objects is stored in some container. A container is a logically or physically contiguous area of secondary storage. There may be zero, one or more containers per physical host. Each container stores a subset of the clusters in the system.

The log-keeping mechanism considers that a cluster is local to a context if its log (see Section 3.2) is accessible in that context. At context termination, all co-located clusters must be deactivated before any one of them may actually be unmapped. This is necessary to ensure that their respective logs can be updated appropriately before their contents are committed to secondary storage ${ }^{1}$.

\subsection{References}

Objects are the vertices and references the edges of the global object graph. Two forms of references are considered: canonical references and language-specific references. Canonical references are used in objects stored on secondary storage and are sent to other contexts. Language-specific references are used between objects co-located within the same context. The process of converting a canonical reference into a language-specific reference is called swizzling; the reverse is called unswizzling.

The log-keeping mechanism relies on the fact that when an object is activated (sometime after its cluster has been mapped into a context), every reference that it contains is swizzled; conversely, when this object is eventually deactivated (before its cluster is unmapped from a context), every (swizzled) references that it contains is unswizzled.

\footnotetext{
${ }^{1}$ This constraint could however be lifted if the local GC could participate in appropriately updating the logs. This would make it possible for the GC to preemptively un-map a cluster which has been deactivated.
} 
Similarly, references are marshalled and unmarshalled when exchanged between contexts. The former involves unswizzling the reference to its canonical form, so that it can be sent across context boundaries, while the latter involves swizzling the reference back to its language-specific form.

The canonical and language specific forms of a reference may in fact be identical. Swizzling and unswizzling may then be null operations, but it is required that every reference crossing a site boundary be examined in turn ${ }^{2}$. However, references exchanged within a context are not trapped by the log-keeping mechanism. This keeps the overhead due to log-keeping to a minimum.

\subsection{Proxies}

When swizzling a reference to an absent object, a proxy for the object is created. If the absent object is already mapped into some other context, a G-proxy is created; such a proxy has the same interface as the remote object that it represents and acts as its surrogate. The G-proxy handles the marshalling and un-marshalling of the parameters to be sent to or received from the remote object that it represents ${ }^{3}$.

If on the other hand the absent object is dormant, i.e., a persistent object stored in some container, a P-proxy for its whole cluster is created ${ }^{4}$. When such an absent object is eventually invoked by some thread of control the entire cluster containing this object is mapped into the current context, overlaying its $\mathrm{P}-$ proxy $^{5}$, and the invoked object is activated.

\subsection{Cross Context Invocations}

The log-keeping mechanism can only be made aware of object invocations made across context boundaries since only these invocations require down-calls to the system, for instance to marshal and unmarshal parameters.

When an object reference is exchanged between a proxy and the server object that it represents, the system is able to identify both the server object and the object to which the reference is being exchanged. The system is however not able to identify the client object since interactions between co-located objects, in this case between the client object and the proxy of the server object, are performed independently from the system.

\subsection{Mature Objects}

Every object is created immature. A global object is promoted, i.e., becomes mature, when a reference to it is marshalled. A persistent object is promoted when a reference to it is unswizzled or when it is first deactivated. The allocation of a global name, or canonical reference, to an object is postponed until it is promoted. When promoted, an object is assigned to a cluster which may have to be created.

\footnotetext{
${ }^{2}$ Except for the special case of the references contained in clusters migrated between containers.

${ }^{3}$ The absent object might eventually be made to overlay its proxy if it is later mapped into the same context. The proxy is thus made to occupy the same amount of space as the object that it represents.

${ }^{4}$ We assume the existence of a mechanism which makes it possible to locate an object for which a reference is known anywhere in the system.

${ }^{5}$ Actually load balancing or security considerations may require that a cluster be mapped in some other context.
} 


\section{Log-Keeping}

This section describes the design of the log-keeping mechanism, its data structures and its algorithm.

\subsection{Notation and Definitions}

The notation introduced below and used throughout the reminder of this paper is only meant to facilitate the description of the mechanism. It does not necessarily reflect the naming scheme of the underlying system.

- $X$ is a cluster (i.e., a name in upper case).

- blue is an object (i.e., a colour name in lower case).

- X.blue is an object blue belonging to cluster $X$.

- $@ A$ is a site which may be either a context or a container (i.e., an upper case letter preceded by an “@”).

- X.blue@ $A$ is an object blue (which belongs to cluster $X$ ) at site $@ A$; $@ A$ being either a container or a context. Note that any of blue or X.blue or X.blue@A can be used interchangeably to refer to the same object.

- $\uparrow$ blue is a reference to the object blue.

- $\{\text { blue }, \cdots, Y, \cdots\}_{X}$ is an entry in the $\log$ of cluster $X$ (see Section 3.2) associating object blue with cluster $Y$. The ellipsis $\cdots$ is used to show that the object may also be associated with other clusters by the same entry as there is at most one entry for a given object.

- $\{\text { blue, } Y\}_{@ A}$ is an entry in the $\log$ of context $@ A$ (see Section 3.5) in this case, @A can only refer to a context since there are no per container log.

\subsection{Clusters As Log-Keeping Unit}

The overall system-wide object graph potentially spans both primary and secondary storage as shown in Figure 2. Keeping information about exchanges of references among objects at the per context or per container level would be difficult not only because contexts are transient entities, but also because of the very dynamic nature of the global object graph. Objects stored in the same container can be dynamically mapped into different contexts, and objects which were at one time co-located in the same context, can eventually be unmapped into different containers or migrated to different contexts. This information should therefore be more closely associated with individual objects.

With an appropriate clustering policy [Gourhant et al., 1992] objects are more likely to reference other objects belonging to the same cluster. Using clusters as log-keeping unit does not only make it possible to share the space overhead of the logs among several clustered objects, but also takes advantage of the locality of reference within clusters. It minimizes the amount of information the log has to keep because exchanges of references between objects belonging to the same cluster do not have to be logged.

Therefore, each cluster maintains a log. Logically, each entry in this log is indexed by the identifier (ID) of some object and contains a list of cluster IDs (and possibly a timestamp). Such an entry means that this object is "known" by each of these associated clusters. A cluster "knows" an object if it either contains a reference to this object, or has an entry in 


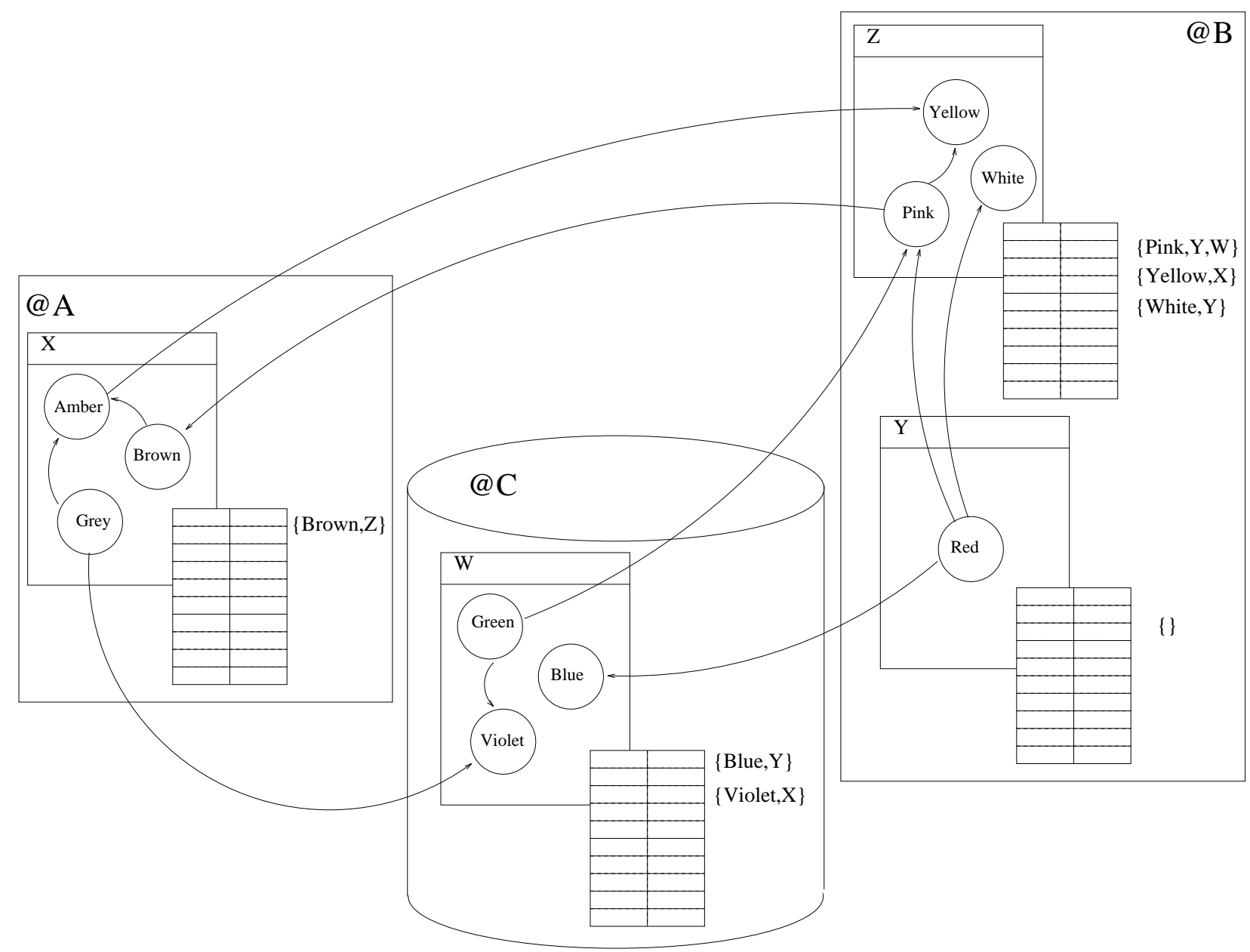

Figure 2: Ideal situation

its log indexed by the ID of this object. The index objects may or may not belong to the cluster where the table resides as will be explained later in Section 3.4.

\subsection{Lazy Log-Keeping}

Figure 2 shows an "ideal" situation whereby every cluster's log shows the complete list of clusters containing references to each of its objects and nothing else. In this Figure, @ $A$ and $@ B$ are contexts while $@ C$ is a container, although, conceptually the distinction does not matter. For instance, object pink in cluster $Z$ mapped in context@B, i.e.. Z.pink@B, is known by Y.red@B and W.green@C. Therefore, the $\log$ of cluster $Z$ contains the entry $\{\operatorname{pink}, Y, W\}_{Z}$.

It should be noted that the global root set of context $a B$ consists of the objects Pink and Yellow. Object White which is only associated with a local cluster in the entry $\{\text { White, } Y\}_{Z}$ does not belong to the global root set.

The situation depicted in Figure 2 is however unlikely to occur if a lazy log-keeping mechanism is used. Such mechanism maintains a weaker invariant that every object in the global root set of some site, has an entry in at least one per-cluster log located at this site. 


\subsection{Partial Back Pointer Path}

The aforementioned invariant can be maintained without necessarily keeping a strictly accurate per cluster $\log$ as shown in Figure 2. It is not necessary for such a log to maintain an exact list of all the clusters holding a reference to some object. However, enough information must be kept to make it possible for the GGD to decide whether or not a particular entry is obsolete and can safely be discarded (see Section 4).

To do so, it must be possible to eventually gather, for every object, the complete list of (not co-located) clusters which contains a reference to this object, even though this list is not necessarily kept entirely in the $\log$ of the cluster to which this object belongs. The idea of this log-keeping mechanism is to lazily leave a trail of partial back pointers along the paths a reference to an object has followed during successive exchanges between sites.

In other words, an entry in a cluster log associates an object with a list of clusters which know this object, where "knowing" may only mean containing an entry indexed by this object in their own log. This entry can be described as a partial back pointer. It is a back pointer because it leads to whatever cluster or clusters know a given object. It is a partial back pointer because it does not point to each individual object which holds such a reference but to their clusters.

In the example shown in Figure 6, an entry in the log of cluster Z, associates an object red with a list of clusters, in this case $\{\text { red, } Y\}_{Z}$. This means that cluster $Y$ contains either a reference $\uparrow$ red, or that its $\log$ contains an entry $\{\text { red, } \cdots\}_{Y}$. The list of clusters which contains a reference to a given object can be gathered by tracing these partial back pointer paths $s^{6}$.

Locality of reference within clusters should therefore contribute to reducing the number of such paths for a given object and hence the complexity of the resulting tree or graph made of the partial back pointer paths rooted at this object.

\subsection{Growth of a Tree of Partial Back Pointer Paths}

This sections describes how a graph of partial back pointer paths grows from the initial entry logged when a newly created object is promoted, up to entries pointing to all the clusters which actually contain a reference to this object. It shows how a root entry can always be logged when a newly created object is promoted and how a reference in transit through a site does not fail to leave behind a partial back pointer path.

Missing Link Cluster: The ID of some cluster, chosen at random among the clusters mapped with a client object is piggy-backed with the parameter list of any remote invocation to a server object in case this invocation may return a reference. This cluster ${ }^{7}$ will be referred to as the missing link cluster.

Per-context log: The per-context $\log$ is a transient structure logically maintained at the context level which persists only for as long as the context. This log logically associates

\footnotetext{
${ }^{6}$ This list does not necessarily include some remote active clusters which may contain a reference to the object. However, since such clusters would be co-located into the same context as some other cluster already logged along these partial back pointer path, the invariant is not broken and an appropriate entry will eventually be logged when their context terminates.

${ }^{7}$ For instance the cluster associated with the ID of the server in the per context log.
} 


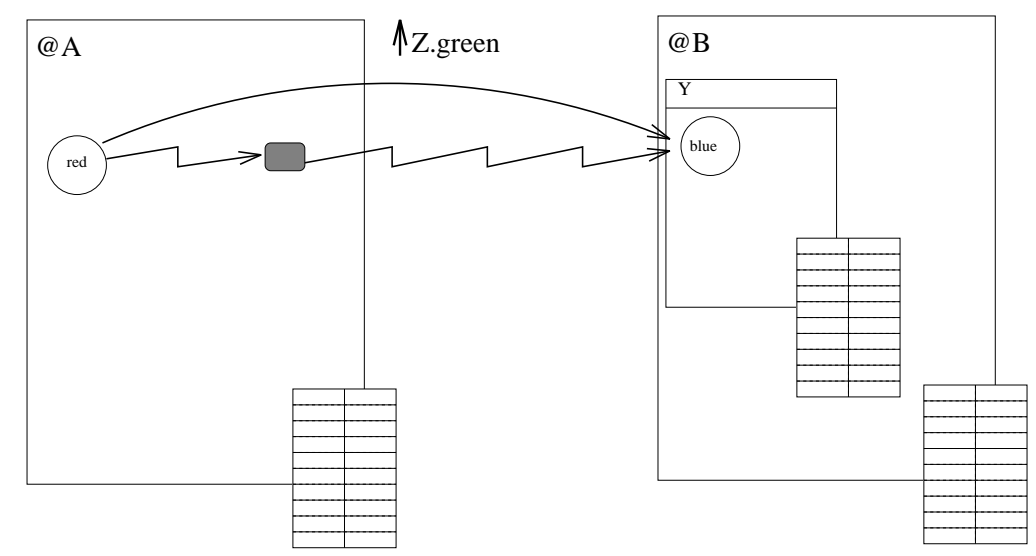

Figure 3: Reference sent as a parameter of a remote invocation

each proxy in the context with the local cluster having first imported (either unmarshalled or swizzled) a reference to this remote object into this context.

Exporting a Reference: A reference to an object can be exported from some context $@ A$ to another context $@ B$ in either of the following two ways:

1. As shown in Figure 3, where a client red@A sends a reference $\uparrow Z$.green to the server Y.blue@B $B^{8}$.

(a) If Z.green is promoted as the result of its reference being marshalled for the first time (see Section 2.7), green has just been allocated to its cluster Z@A. The identity of the remote server Y.blue@B being known (see Section 2.6), the entry $\{\text { green, } Y\}_{Z}$ must be logged ${ }^{9}$.

(b) The entry $\{\text { green }, \cdots, Y, \cdots\}_{Z}$ must also be logged if Z.green was already mature and mapped into context $@ A$.

(c) If Z.green is not mapped in context@A, an entry indexed by green necessarily exists in the per context log. For instance if this entry is $\{\text { green, } W\}_{@ A}$, the entry $\{\text { green }, \cdots, Y, \cdots\}_{W}$ must be logged.

2. Figure 4 shows a server $X$.red@A returning a reference $\uparrow Z$.green to client blue@B as the result of some invocation. As the identity of the remote client is not known (see Section 2.6) the ID of the missing link cluster, for instance $V @ B$, is used instead.

(a) If Z.green is promoted as the result of its reference being marshalled for the first time (see Section 2.7), green has just been allocated to its cluster Z@A. The root entry $\{\text { green, } V\}_{Z}$ must be logged.

(b) Similarly, if Z.green was already mature and mapped into context @ $A$, the entry $\{\text { green, } \cdots, V, \cdots\}_{Z}$ must logged.

(c) If Z.green is not mapped in context@A, an entry indexed by green necessarily exists in the per context log. For instance if this entry is $\{\text { green, } W\}_{@ A}$, the entry $\{\text { green, } \cdots, V, \cdots\}_{W}$ must be logged.

\footnotetext{
${ }^{8}$ The proxy of the server is represented as a small grey square.

${ }^{9}$ An entry is only logged into some table if it is not already present.
} 


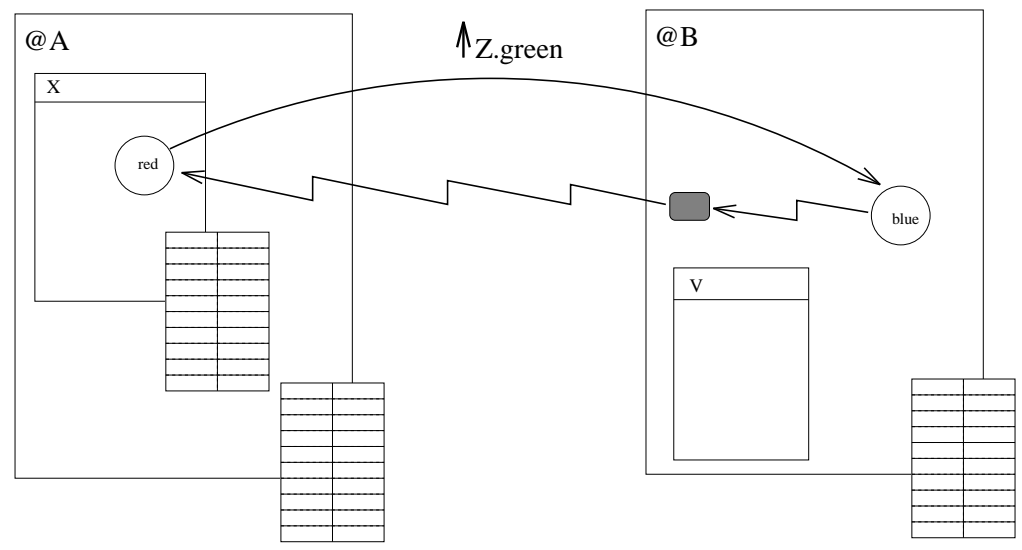

Figure 4: Reference returned as a result of a remote invocation

When a global object is promoted, that is, when its reference crosses a site boundary for the first time (marshalled), an entry for this object can always be logged immediately into the $\log$ of its cluster. This entry constitutes the first link, or root entry, in a partial back pointer path rooted at this object. From this point, and until the GGD eventually removes this entry, if ever (see Section 4), and as long as this entry is associated to some non-local clusters, local per site GCs will consider this object as a global root.

Furthermore, when a reference to either a local mature object, or a reference to some remote object is exported, the log of respectively the cluster to which this object belongs, or the cluster known to have initially imported the reference into the context can be updated with an entry pointing to the next link in the partial back point path.

Importing a Reference: A reference to an object can be imported into some context $@ B$ from another context $@ A$ in either of the two ways previously described. The object to which the reference is imported is already mature.

1. As shown in Figure 3, where the server Y.bluє@B receives a reference $\uparrow Z$.green from the client red@A.

If Z.green is not mapped in $Q B B$ and there is no entry indexed by green in the $\log$ of context $@ B$, the entry $\{$ green, $Y\} @ B$ must be logged.

2. As shown in Figure 4, where the client blue@B receives a reference $\uparrow Z$.green from a server X.red@A as the result of some invocation. The identity of the client, i.e., of the object who first imports the reference into context $@ B$, is not known (see Section 2.6). The ID of the missing link cluster, for instance $V @ B$, must be used instead.

If Z.green is not mapped in @B, and there is no entry indexed by green in the $\log$ of context@B, the entry $\{\text { green, } V\}_{® B}$ must be logged.

When a remote reference is imported for the first time, i.e., as soon as a proxy is created for the global object to which the reference is imported, an entry is logged in the per context log. The cluster associated with this entry is identical to the cluster associated with the corresponding entry in the per-cluster log of the object to which the reference is being imported. 
Activating a Cluster: When some cluster $X$ is activated into context $@ A$, for each reference to some non-local object which is swizzled but not yet indexed in the log of @ $A$, e.g., $\uparrow Z$.green, the entry $\{\text { green, } X\}_{@ A}$ must be logged.

Additionally, if activating a cluster results in overlapping a proxy, i.e., if an object which was indexed in the per-context $\log$ is mapped, the corresponding entry in the per-context $\log$ must be removed. Activating a cluster is equivalent to importing all the references that it contains.

De-activating a Cluster: When some cluster $X$ is deactivated from context@A, for each remote reference being unswizzled, the cluster indexed by the referenced object in the per-context $\log$ must be updated.

For instance, when $\uparrow Z$.green is unswizzled, if the entry indexed by green in the per-context $\log$ is $\{\text { green, } W\}_{@ A}$, the entry $\{\text { green }, \cdots, X, \cdots\}_{W}$ must be logged.

If unswizzling $\uparrow Z$.green actually results in promoting object $Z$.green, which would then necessarily be local, the root entry $\{\text { green, } X\}_{Z}$ must be logged.

When a reference to a persistent object is first unswizzled, i.e., when a persistent object is promoted because its reference is held by a cluster being deactivated, the root entry for this object can be logged directly in the $\log$ of its cluster ${ }^{10}$. Similarly an entry associating the cluster being deactivated with a local mature object to which a reference is unswizzled can also be logged directly. Entries associating some remote object with the cluster being deactivated are however logged into the $\log$ of the cluster known to have first imported it (found in the per-context log).

Even though inter-cluster but intra-context exchanges of references cannot be trapped by the log-keeping mechanism, they are eventually logged when clusters are deactivated. Deactivating a cluster is equivalent to exporting all the references that it contains.

References in transit: Whenever a reference to some object transits through a context, it is first logged into the log of this context, associated with the cluster known to have first imported this reference. This cluster can either be the cluster having actually imported it, or a missing link cluster arbitrarily chosen. What matters is that both parties involved in exchanging a reference agree upon which cluster is known to have imported it. In this way, the previous link in the partial back pointer path points to this cluster. And the log of this cluster may eventually become the next link in the path, should this reference be re-exported to another site. The path of partial back pointers therefore remains unbroken. This is illustrated in the following example:

Figure 5 shows an object pink@B which invokes object Y.blue@A. Y.blue@A returns the reference $\uparrow Y$.green@A as the result of this invocation. The identity of the cluster of the client object (in this case pink) is not known ${ }^{11}$, the missing link cluster $V @ B$ is used instead so that the entries $\{\text { green, } V\}_{Y}$ and $\{\text { green, } V\}_{® B}$ can be logged. Object pink@B $B^{12}$ later re-exports this reference by invoking object X.amber@C and passes the reference $\uparrow Y$.green@A as one parameter of this invocation. The cluster of the remote server, i.e., $X$, being known, the entries $\{\text { green, } X\}_{V}$ and $\{\text { green, } X\}_{® C}$ are logged.

\footnotetext{
${ }^{10}$ When a persistent object is promoted by crossing a site boundary, that is, when this object is itself deactivated, no per-cluster log needs to be updated.

${ }^{11}$ Furthermore, pink may not be mature.

${ }^{12}$ It could be any other object mapped in context @B.
} 


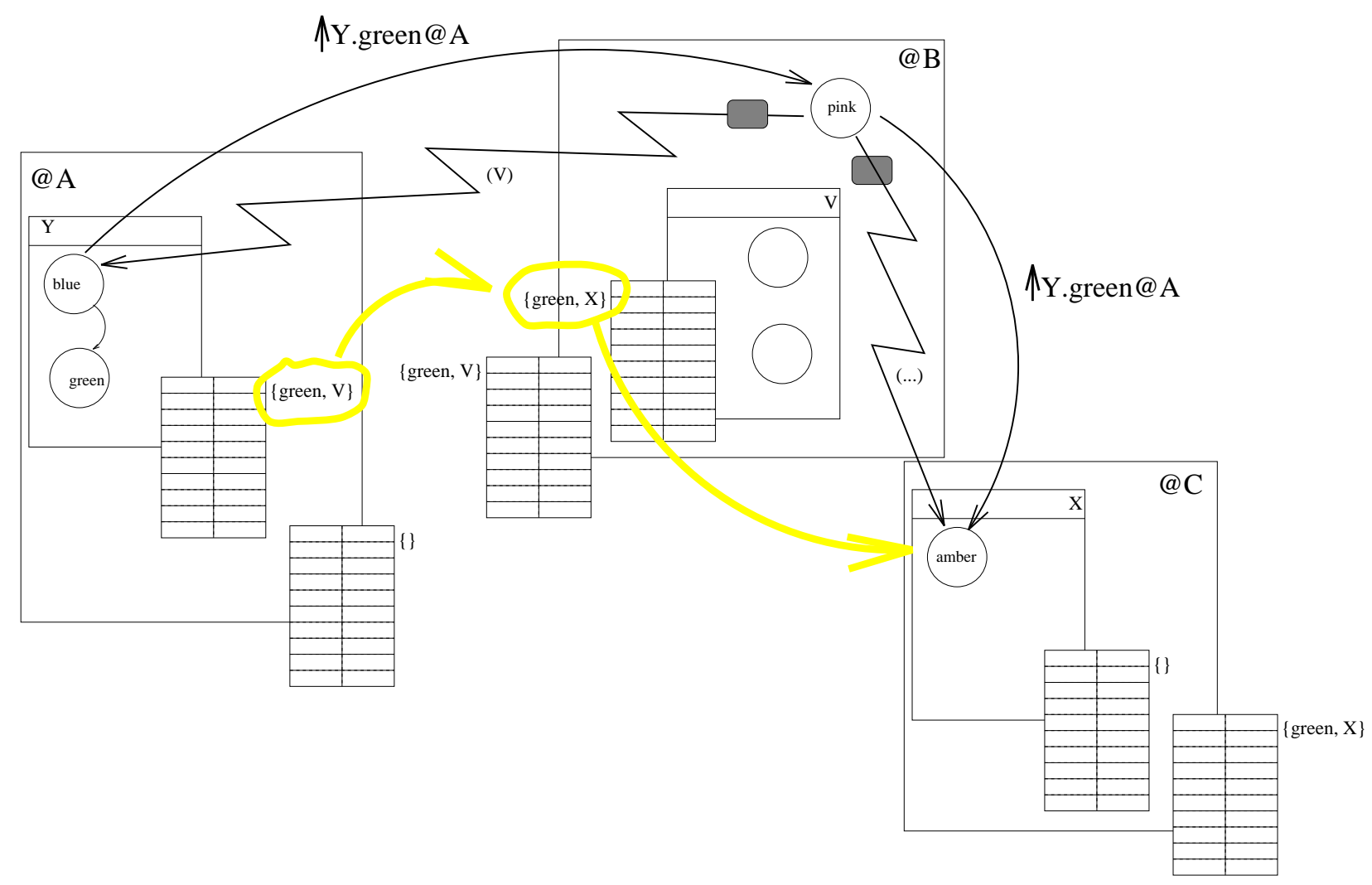

Figure 5: A reference in transit

\subsection{Inaccuracies in the Logs}

Figure 6 represents a set of clusters and the contents of their respective logs as could be observed after a few exchanges of references have taken place within the system. Sites boundaries are not represented. Unlike the "ideal" situation represented in Figure 2, their logs are not necessarily accurate, although they contain enough information for any local garbage collector to be safe, no matter how these clusters eventually end up being distributed across different sites. In other words, although these logs may contain inaccuracies, they are nevertheless complete, since the invariant stated in Section 3.3 is not broken.

These logs may contain three kind of inaccuracies:

- Obsolete entries such as $\{\text { blue, } W\}_{Z}$ and $\{\text { maroon, } Y\}_{Z}$. The former is obsolete because the reference to object blue previously held by some object of cluster $W$ does not exist anymore; the latter because object maroon does not exist anymore. Both entries would eventually be removed by the GGD.

- Incomplete entries, i.e., which give an incomplete list of the clusters actually containing a reference to some object. This list can nevertheless be reconstructed using available information held by other per-cluster logs which form the partial back pointer path.

For instance, the entry like $\{\text { red }, W, X\}_{Z}$ would be more accurate than the entry $\{r e d, Y\}_{Z}$ that the per-cluster $\log$ for $Z$ actually contains. However the partial back pointer path can be traced as follow: entry $\{\text { red, } Y\}_{Z}$, means that object red is known to cluster $Y$. However, no object in cluster $Y$ holds any reference to object red, but 


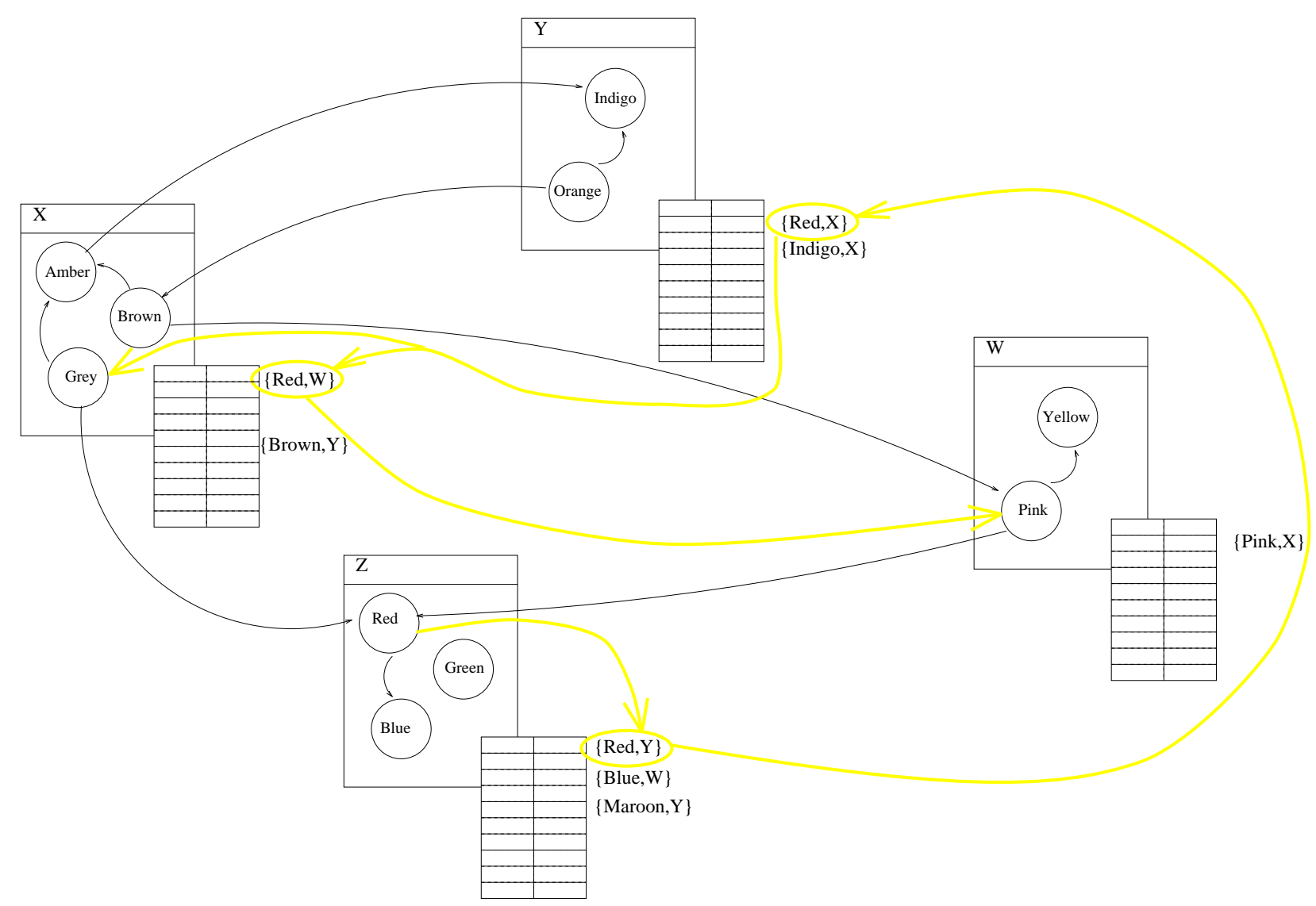

Figure 6: Partial back pointer path

there is an entry $\{\text { red, } X\}_{Y}$. In turn object $X$.grey actually holds a reference $\uparrow Z$.red and there is an entry $\{r e d, W\}_{X}$ in the same cluster. In cluster $W$, object W.pink is the only place where a reference $\uparrow Z$.red can be found.

- Entries belonging to some partial back pointer path such as $\{\text { red, } Y\}_{Z}$. Although no objects in cluster $Y$ per se holds any reference to object Z.red, this entry is not obsolete since the $\log$ of cluster $Y$ contains an entry indexed by object red.

$Y$ may either have been used as a missing link cluster, or an object in $Y$ may have once held the reference $\uparrow Z$.red and forgotten it after having forwarded it to X.grey, or else, $Y$ may have been the first cluster to have imported $\uparrow Z$.red in a context where it was mapped although it was another cluster which re-exported it to X.grey. It is not possible at this stage to know which of these possibilities applies, which shows, as stated in Section 3.5, that any cluster is equally valid to serve as the missing link cluster. Similarly, the entry $\{\text { red, } X\}_{Y}$ is not obsolete either even though this cluster does not contain any object red.

\section{Pruning the Partial Back Pointer Path Trees}

The rôle of the GGD is to identify which entries in the different logs maintained by the log-keeping mechanism, i.e., per-cluster logs and per-context Any comprehensive, i.e., graphtracing, GGD policy can be used. See Louboutin and Cahill [Louboutin and Cahill, 1995] 
for further details about an adaptation of an algorithm for GGD inspired by that of Schelvis [Schelvis, 1989] using this lazy per-cluster log-keeping mechanism.

Garbage Objects: An object becomes garbage when it is not reachable from any local root and there is no entry in the log of any local cluster associating the object's ID with the ID of some non-local cluster.

Garbage Clusters: A cluster becomes garbage when it contains only garbage objects, and there is no entry in its per-cluster log associating some object's ID with the ID of some non-local cluster.

Garbage per-cluster log entry: An entry in some per-cluster log indexed by some object red can be collected when red is no longer "known" by any of the associated clusters in this entry. An entry can also be collected whenever the object indexing it has been collected by the GC.

Garbage per-context log entry: An entry in some per-context log indexed by some object blue can be collected by the local per-context $\mathrm{GC}^{13}$ when no local object contains any reference to object blue, i.e., as soon as the proxy for blue is itself collected by the local GC, or when the actual object blue eventually overlays its own proxy $^{14}$

\section{$5 \quad$ Related Work}

Ferreira and Shapiro [Ferreira and Shapiro, 1994] describe a system based on a Distributed Shared Memory (DSM) model rather than the Remote Procedure Call (RPC)/objectswapping model adopted by Amadeus; it features fine-grain, i.e., smaller than a page, objects, clustered into fixed-size, i.e., made of a fixed number of contiguous pages, and disjoint, "segments." These segments are themselves logically grouped into "bunches." Bunches can be replicated and shared via the underlying weakly consistent DSM system. Objects are identified by their address within a 64 bit system-wide address space encompassing both primary and secondary storage $\mathrm{e}^{15}$. GC is performed at two levels; a per bunch comprehensive GC and a "scion cleaner" which is not comprehensive. A heuristic is used to group bunches at one site so that a comprehensive GC can tackle cycles locally.

This approach relates to ours in the sense that both systems use some form of object clustering, and that log-keeping is done on a per cluster basis. However, when an inter-bunch reference is created ${ }^{16}$ and the bunch of the target object is not local, a "scion-message" must eventually be sent to the target; this creates an additional overhead (additional message) for mutator processes. Race conditions that these additional log-keeping messages would create are avoided by piggy-packing these messages on the messages used by the underlying consistency protocol. Our system avoids such additional log-keeping messages altogether.

\footnotetext{
${ }^{13}$ Unlike entries in per-cluster logs which can only be collected by the GGD mechanism.

${ }^{14}$ The per-context log is not implemented as an independent data structure but as an additional field in the header of an object proxy (see Section 2.5) containing the ID of the associated cluster.

${ }^{15}$ Object addressing is a combination of OID and SSP approaches [Plainfossé, 1994a].

${ }^{16}$ Such creation is trapped via a "write-barrier" unlike our RPC based system which uses (un)swizzling operations.
} 


\section{Conclusion}

Laziness: Our log-keeping mechanism does not attempt to update remote third party logs even in the case of exchanges of third party references, it does not require additional "control" messages, and hence avoids race conditions common to eager log-keeping approaches. This mechanism also postpones the update of the $\log$ as late as possible (e.g., until context termination for inter-cluster, intra-context exchanges of references), hence, it does not trigger object-faults which would have not otherwise occurred.

Log-keeping for comprehensive GGD: The first time a reference to some target object crosses a site boundary, an appropriate entry can always be logged in the target's cluster. This initial partial back-pointer identifies the target as a global root. Our mechanism ensures that when this reference subsequently crosses another site boundary, that there is a co-located cluster, already belonging to the partial back-pointer path, where an appropriate entry can be logged. Thus it can be seen that the complete list of the clusters which hold a reference to a given object can be gathered by transitively tracing these partial back-pointer paths. It therefore maintains enough information for a comprehensive GGD which would proceed by tracing the graphs of partial back-pointer paths rather than the actual object graph.

Robustness: This mechanism is robust. Logs are updated when a reference is marshalled, unmarshalled, unswizzled or swizzled, and before such action is actually performed. If the actual exchange of reference or the mapping or unmapping of some cluster which triggered these log-keeping operations fails, it would result in some unnecessary log entries which would have to be later collected. It would result in additional detection latency but would not affect the safety property of the GGD.

Overhead: The overhead of this mechanism is mostly space overhead, i.e., size of the per-cluster logs and possibly clusters containing only garbage objects but which cannot be collected. Since log-keeping operations are performed by trapping (un)swizzling operations it should only add a negligible computing overhead.

However, a GGD using this log-keeping mechanism generates potentially more inter-site messages than a GGD which traces the actual object graph or uses an eager log-keeping mechanism. These additional messages are due to the inaccuracies in the logs explained in Section 3.6. We contend that shifting the overhead from the log-keeping mechanism, i.e., from the mutator processes, to the GGD is in itself beneficial to overall system performance even if it does not decrease the number of messages exchanged globally.

The mechanism requires that some information (of the size of a cluster ID) be piggybacked on the parameter lists of all inter-context object invocations. This overhead is deemed acceptable.

Worst case scenario: If $k$ is the total number of objects in the system, and $n$ the total number of clusters in the system, a worst case scenario may generate a per-cluster log of size $k(n-1)$, i.e., a monstrous per-cluster $\log$ with $k$ entries, each of them associated with $n-1$ cluster IDs. If every per-cluster log in the system grow to this proportion, a potential space overhead of $k n(n-1)$ sizeof $(I D)$ would have to be considered.

However, it is expected that such a scenario is highly unlikely to occur, and that typical cases would be more reasonable due to object clustering (and dynamic re-clustering) and locality of reference within clusters, and among clusters. An effective GGD algorithm would 
also contribute to continuously keeping the growth of the logs under control. The log-keeping mechanism could also be optimized so as not to log entries corresponding to exchanges of references between clusters which remain co-located after being (un-)mapped.

\section{References}

[Bevan, 1987] Bevan, D. (1987). Distributed Garbage Collection using Reference Counting. PARLE (Parallel Architectures and Languages Europe), pages 176-187. LNCS 259.

[Cahill et al., 1993] Cahill, V., Baker, S., Starovic, G., and Horn, C. (1993). Generic runtime support for distributed persistent programming. In Paepcke, A., editor, OOPSLA (Object-Oriented Programming Systems, Languages and Applications) '93 Conference Proceedings, volume 28, pages 144-161, Washington D.C., USA. ACM, New York. Also technical report TCD-CS-93-37, Dept. of Computer Science, Trinity College Dublin. ftp://ftp.dsg.cs.tcd.ie/pub/doc/TCD-CS-93-37.ps.gz.

[Dickman, 1991] Dickman, P. W. (1991). Distributed Object Management in a Non-Small Graph of Autonomous Networks with Few Failures. PhD thesis, Darwin College, Cambridge University.

[Ferreira and Shapiro, 1994] Ferreira, P. and Shapiro, M. (1994). Garbage collection and DSM consistency. In Proceedings of the First Symposium on Operating Systems Design and Implementation (OSDI), Monterey, California, USA.

[Gourhant et al., 1992] Gourhant, Y., Louboutin, S., Cahill, V., Condon, A., Starovic, G., and Tangney, B. (1992). Dynamic Clustering in an Object-Oriented Distributed System. In Proceedings of OLDA-II (Objects in Large Distributed Applications), Ottawa, Canada. ftp://ftp.dsg.cs.tcd.ie/pub/doc/dsg-24.ps.gz.

[Louboutin and Cahill, 1995] Louboutin, S. R. and Cahill, V. (1995). On Comprehensive Global Garbage Detection. In European Research Seminar on Advances in Distributed Systems (ERSADS), Alpes d'Huez, France. Also technical report TCD-CS-95-11, Dept. of Computer Science, Trinity College Dublin. ftp://ftp.dsg.cs.tcd.ie/pub/doc/TCD-CS95-11.ps.gz.

[Plainfossé, 1994a] Plainfossé, D. (1994a). Comparaisons entre les OIDs et les chaînes de PSS. Note Technique SOR-131, INRIA-SOR, Paris, France.

[Plainfossé, 1994b] Plainfossé, D. (1994b). Distributed Garbage Collection and Referencing Management in the Soul Object Support System. PhD thesis, Université Pierre \& Marie Curie - Paris VI, Paris, France.

[Schelvis, 1989] Schelvis, M. (1989). Incremental Distribution of Timestamp Packets: A New Approach To Distributed Garbage Collection. In Proceedings OOPSLA'89, pages 37-48, New Orleans. ACM.

[Watson and Watson, 1987] Watson, P. and Watson, I. (1987). An efficient garbage collection scheme for parallel computer architectures. In de Bakker, J., Nijmaan, A., and Treleaven, P., editors, PARLE (Parallel Architectures and Languages Europe), pages 432443, Eindhoven, The Netherlands. 